\title{
Iatrogenic Full-Length Intramural Esophageal Dissection Associated with Pneumomediastinum after Attempted Diagnostic Gastroscopy
}

\section{Chin-Ho Lee ${ }^{1}$, Wen Pin Chen ${ }^{1}$, Yu-Min Feng ${ }^{2}$ and Pei-Yu Huang ${ }^{3}$}

${ }^{1}$ Department of Medical Imaging, Chia-Yi Christian Hospital, Taiwan

${ }^{2}$ Division of Gastroenterology and Hepatology, Department of Internal Medicine, Chia-Yi Christian Hospital, Taiwan

${ }^{3}$ Department of Radiology, Kaohsiung Municipal Min-Sheng Hospital, Taiwan

\begin{abstract}
Upper gastrointestinal endoscopy is a usually safe procedure. Intramural Esophageal Dissection (IED) is a rare disorder often due to the abrupt increased intraesophageal pressure after given the existence of mucosal tear. We present an extremely rare case of iatrogenic IED complicated with pneumomediastinum after a failed gastroscopy for healthy person. Reformatted computed tomographic images successfully established the diagnosis by means of the esophageal double lumen as full extent of intervening mucosal flap and mediastinal air. The patient got improvement following appropriate conservative management. Our case adds support of the hypothesis of gas diffusion across the muscular layers of the esophagus rather than real defect.
\end{abstract}

Keywords: Gastroscopy; Esophagus; Intramural dissection; Perforation; Pneumomediastinum

\section{Introduction}

Routine Esophageal Gastroendoscopy (EGD) is generally a safe procedure as we know. Esophageal laceration with intramural dissection is an uncommon injury demonstrating esophageal separation of mucosa and/or submucosa from deeper muscular layers, often due to the abrupt increased intraesophageal pressure. Progression to esophageal perforation is extremely rare as reported. We present a case developing Intramural Esophageal Dissection (IED) combined with extraluminal air leakage after an attempted diagnostic gastroscopy, in which chest Computed Tomography (CT) successfully established a diagnosis. Our patient recovered completely with conservative management.

\section{Case Report}

A middle-aged female patient received health gastroendoscopy. She denied any underlying disease, though sometimes acid regurgitation and heartburn sensation were mentioned. The EGD was performed by an experienced and senior gastroenterologist. However, the scope could not pass through the inlet of esophagus without excessive force reported; the procedure failed under suspicion of cricopharyngeal spasm. A few hours later, the patient came back to the hospital due to the complaint of sore throat, vomiting, and subsequently chest pain. An electrocardiogram showed no abnormal finding. There also was no relevant abnormality detected on the initial interpretation of chest radiograph. Chest CT scan (Figures 1a-c) was subsequently arranged and revealed full-length, circumferential intramural dissection of the esophagus manifesting the esophageal double lumen as air-distended false and collapsed true lumen, and small amounts of air in the paraesophageal space. The collapsed true lumen made it difficult to find subtle mucosal tear. The diagnosis of IED associated with pneumomediastinum was established on the basis of imaging findings.

Because there were no clinical sign of sepsis and the study demonstrated the mildest form of esophageal perforation, the patient got conservative management consisting of broad spectrum intravenous antibiotics, pain control, nothing by mouth, and total parenteral nutrition. When her general condition became stable with a tolerance of liquid diet, she was discharged on day 7 , and had uneventful OPD follow-up. The esophagogram with barium (Figure 1d) was carried out two months later, and there was no evidence of contrast extravasation or luminal narrowing.

\section{Discussion}

Upper gastrointestinal endoscopy has now been conventional medical practice after developing the flexible fiberoptic endoscope, is generally a safe procedure. A large study in pediatrics revealed incidences of mucosal tear and esophageal perforation were 2.14 and 1.07 , separately, per 10000 procedures [1].

Intramural esophageal dissection (IED), a rare disorder, was first mentioned in 1968 by Marks and Keet [2]. IED is initiated by a mucosal tear accompanying with a creation of a true and false lumen in the esophagus, conceptually similar to an aortic dissection [3]. It is generally considered that the most common causes of IED are iatrogenic, including instrument insertion (gastroendoscopy, nasogastric tube, and transesophageal echocardiography) and hemostatic applications (variceal sclerotherapy and argon-plasma coagulation), as well as noniatrogenic causes (ingestion or impaction of foreign bodies) or spontaneously [4]. The first case of iatrogenic IED after attempted diagnostic gastroendoscopy was described in 2009 [5] with possible risk factors including direct injury by the tip of the endoscope, barotraumas from air insufflation during attempted intubation, or cricopharyngeal spasms; as we met.

The imaging documented full extent of circumferential intramural dissection of the whole esophagus was very rare [6]. In addition, only few cases associated with esophageal perforation were noted $[3,4,6-9]$, which firstly reported coexistence of IED and extraluminal air leakage in 2008 [4]. Though, Monu and Murphy [3] thought that previously reported cases of IED that did not use CT might be underestimated and only able to exclude more serious esophageal perforations with

*Corresponding author: Pei-Yu Huang, Department of Radiology, Kaohsiung Municipal Min-Sheng Hospital, No. 134, Kaisyuan 2nd Rd. 802 Kaohsiung City Taiwan, Tel: +886-7-7511131-2145; E-mail: pyhung0506@yahoo.com.tw

Received September 10, 2014; Accepted October 24, 2014; Published October 26, 2014

Citation: Lee CH, Chen WP, Feng YM, Huang PY (2014) latrogenic Full-Length Intramural Esophageal Dissection Associated with Pneumomediastinum after Attempted Diagnostic Gastroscopy. J Clin Case Rep 4: 443. doi:10.4172/21657920.1000443

Copyright: @ $2014 \mathrm{Lee} \mathrm{CH}$, et al. This is an open-access article distributed under the terms of the Creative Commons Attribution License, which permits unrestricted use, distribution, and reproduction in any medium, provided the original author and source are credited. 
Citation: Lee CH, Chen WP, Feng YM, Huang PY (2014) latrogenic Full-Length Intramural Esophageal Dissection Associated with Pneumomediastinum after Attempted Diagnostic Gastroscopy. J Clin Case Rep 4: 443. doi:10.4172/2165-7920.1000443

Page 2 of 2

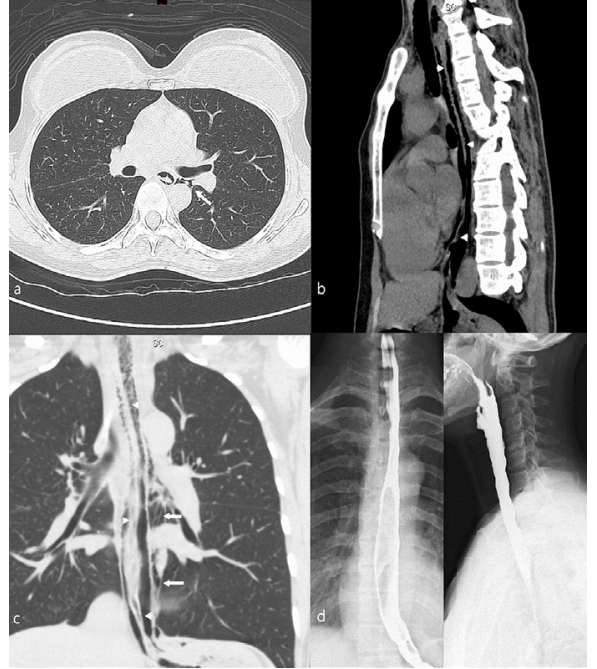

Figure 1: A middle-aged woman with intramural dissection of esophagus. Axial (a) and Curved Multi-planar Reconstruction (MPR) sagittal (b) and coronal images (c) from chest CT with lung window setting showed esophageal double lumen. (a) Axial CT image shows a submucosal dissection with circumferential false lumen and small amounts of posterior mediastinal extraluminal air (arrow). (b,c) Sagittal and coronal reformatted images provided depiction of the true and false lumen separated by intervening mucosal flap (arrowheads); and full extent of double lumen of whole esophagus with pneumomediastinum (arrows) in single image plane. The extraluminal air located in the middle, lower thoracic paraesophageal spaces. (d) Barium swallow images two months later revealed no evidence of extraluminal contrast leakage, luminal stenosis. Besides, no abnormal air collection in the central shadow was found.

obvious contrast leakage or large amounts of pneumomediastinum using contrast esophagography.

Review of existing English literature, our case was the first description of IED complicated with pneumomediastinum after an attempted diagnostic gastroendoscopy for healthy person. Unlike the other iatrogenic insult [4] during therapeutic endoscopy for removal of impacted food material with air expanding, perhaps induced extravasation of insufflated air into the mediastinum after mucosal injury with intramural dissection developed. In our case, similar to spontaneous one, given the existence of mucosal tear complicated by endoscopy was initiated; a subsequent increase in pressure within esophagus such as retching or vomiting then potentiated a separation of mucosa and/or submucosa from the deeper muscular layers.

Advanced CT images including volumetric data acquisition allowing for multi-dimensional reformatting is the ideal investigative modality with high specificity for an accurate diagnosis, and will likely have an important role while simultaneously assessing for more serious conditions like esophageal rupture and aortic dissection. In CT imaging, the wall structure of a hollow organ can be best-visualized and analyzed using short axis plane (i.e., axial images), nevertheless reformatted sagittal and coronal images are useful to grasp the extent of the esophageal dissection in the long-axis direction and to detect a relatively smaller hole in the wall of esophagus.

IED complicated with a small amount of pneumomediastinum was considered the mildest form of esophageal perforation [3,10]. Besides, in contrast to the rest of the gastrointestinal tract, the esophagus is not protected by a serosal layer. We thought that our case adds support of the hypothesis [4] of gas diffusion across the muscular layers of the esophagus rather than real defect at the esophageal wall because of a failed gastroscopy and no extraluminal air around proximal esophagus detected. So the conservative treatment is possible as management of classical IED.

Based on clinically stable condition of the patient and aforementioned statement, supportive treatments with nil per oral, pain control, intravenous antibiotics and parenteral nutrition were successfully pursued.

\section{Conclusion}

In summary, we report an unusually complicated case of iatrogenic IED that involved full-length of the esophagus, combined with pneumomediastinum. In patients with chest pain after endoscopy, esophageal injury should be always taken into consideration. As our case, chest CT enabled the diagnosis with the use of reconstructive techniques as curved MPR. The mildest form of esophageal perforation, like the few reported instances $[3,4,9]$, could heal without sequela spontaneously after conservative management.

\section{References}

1. Iqbal CW, Askegard-Giesmann JR, Pham TH, Ishitani MB, Moir CR (2008) Pediatric endoscopic injuries: incidence, management, and outcomes. J Pediatr Surg 43: 911-915.

2. Marks IN, Keet AD (1968) Intramural rupture of the oesophagus. Br Med J 3 : 536-537.

3. Monu NC, Murphy BL (2013) Intramural esophageal dissection associated with esophageal perforation. R I Med J (2013) 96: 44-46.

4. Soulellis CA, Hilzenrat N, Levental M (2008) Intramucosal esophageal dissection leading to esophageal perforation: case report and review of the literature. Gastroenterol Hepatol 4: 362-365

5. Tang SJ, Tang L, Jazrawi SF, Meyer D, Wait MA, et al. (2009) latrogenic esophageal submucosal dissection after attempted diagnostic gastroscopy (with videos). The Laryngoscope 119: 36-38

6. Wang SH, Ruan Z, Liu FB, Huang HL, Zheng J, et al. (2011) A rare case of circumferential intramural dissection of thoracic esophagus with contained esophageal perforation. Chin Med J (Engl) 124: 3433-3435.

7. Zhu RY, Law TT, Tong D, Tam G, Law S (2014) Spontaneous circumferential intramural esophageal dissection complicated with esophageal perforation and esophageal-pleural fistula: a case report and literature review. Dis of the esophagus.

8. Liguori G, Cortale M, Cimino F, Sozzi M (2008) Circumferential mucosa dissection and esophageal perforation in a patient with eosinophilic esophagitis. World J Gastroenterol 14: 803-804.

9. Cha IH, Kim JN, Kwon SO, Kim SY, Oh MK, et al. (2012) A case of conservatively resolved intramural esophageal dissection combined with pneumomediastinum. Korean J Gastroenterol 60: 249-252.

10. Skinner DB, RHR B (1988) Instrumental perforation and mediastinitis. Management of Esophageal Disease: 783. 